\title{
Targeting Bacterial Gyrase with Cystobactamid, Fluoroquinolone, and Aminocoumarin Antibiotics Induces Distinct Molecular Signatures in Pseudomonas aeruginosa
}

\author{
(D) Raimo Franke, ${ }^{a}$ Heike Overwin, ${ }^{a}$ (D) Susanne Häussler, ${ }^{b, c}$ (D) Mark Brönstrupa,d \\ aDepartment of Chemical Biology, Helmholtz Centre for Infection Research, Braunschweig, Germany \\ bInstitute of Molecular Bacteriology, Twincore, Centre for Clinical and Experimental Infection Research, Hannover, Germany \\ 'Department of Molecular Bacteriology, Helmholtz Centre for Infection Research, Braunschweig, Germany \\ dGerman Centre for Infection Research (DZIF), Braunschweig, Germany
}

\begin{abstract}
The design of novel antibiotics relies on a profound understanding of their mechanism of action. While it has been shown that cellular effects of antibiotics cluster according to their molecular targets, we investigated whether compounds binding to different sites of the same target can be differentiated by their transcriptome or metabolome signatures. The effects of three fluoroquinolones, two aminocoumarins, and two cystobactamids, all inhibiting bacterial gyrase, on Pseudomonas aeruginosa at subinhibitory concentrations could be distinguished clearly by RNA sequencing as well as metabolomics. We observed a strong (2.8- to 212-fold) induction of autolysistriggering pyocins in all gyrase inhibitors, which correlated with extracellular DNA (eDNA) release. Gyrase B-binding aminocoumarins induced the most pronounced changes, including a strong downregulation of phenazine and rhamnolipid virulence factors. Cystobactamids led to a downregulation of a glucose catabolism pathway. The study implies that clustering cellular mechanisms of action according to the primary target needs to take class-dependent variances into account.
\end{abstract}

IMPORTANCE Novel antibiotics are urgently needed to tackle the growing worldwide problem of antimicrobial resistance. Bacterial pathogens possess few privileged targets for a successful therapy: the majority of existing antibiotics as well as current candidates in development target the complex bacterial machinery for cell wall synthesis, protein synthesis, or DNA replication. An important mechanistic question addressed by this study is whether inhibiting such a complex target at different sites with different compounds has similar or differentiated cellular consequences. Using transcriptomics and metabolomics, we demonstrate that three different classes of gyrase inhibitors can be distinguished by their molecular signatures in $P$. aeruginosa. We describe the cellular effects of a promising, recently identified gyrase inhibitor class, the cystobactamids, in comparison to those of the established gyrase A-binding fluoroquinolones and the gyrase B-binding aminocoumarins. The study results have implications for mode-of-action discovery approaches based on target-specific reference compounds, as they highlight the intraclass variability of cellular compound effects.

KEYWORDS antibiotics, Pseudomonas aeruginosa, mode of action, gyrase, metabolomics, RNA sequencing, DNA gyrase

- he rise of antimicrobial resistance (AMR) has been identified as a serious threat for global health (1). The number of novel antibiotics in clinical development to fight AMR is small, and most of them represent improved versions of established drug classes. To acknowledge "innovation," antibiotic pipeline reports highlight those belonging to a

Citation Franke R, Overwin H, Häussler S, Brönstrup M. 2021. Targeting bacterial gyrase with cystobactamid, fluoroquinolone, and aminocoumarin antibiotics induces distinct molecular signatures in Pseudomonas aeruginosa. mSystems 6:e00610-21. https://doi .org/10.1128/mSystems.00610-21. Editor Matthew F. Traxler, University of California, Berkeley

Copyright $\odot 2021$ Franke et al. This is an openaccess article distributed under the terms of the Creative Commons Attribution 4.0 International license. Address correspondence to Mark Brönstrup, Mark.Broenstrup@helmholtz-hzi.de.

Received 18 May 2021

Accepted 22 June 2021

Published 13 July 2021 
new structural class and those with a new mechanism of action (2). However, the latter classification into new versus old is not clear-cut: for example, does an antibiotic that addresses a novel binding site on bacterial ribosomes and that causes a distinct arrest of translation fall into "new" or "old"? A medically relevant criterion concerns the ability of the new agent to break resistance, whereas a more mechanistic perspective looks at overall cellular consequences of antibiotic treatments, e.g., on the cellular transcriptome or metabolome $(3,4)$. A series of recent elegant studies has demonstrated that antibiotics addressing different cellular targets induce differential responses on the metabolome or transcriptome (5-9). Moreover, the typical response pattern can serve to assign the mode of action of novel antibacterial compounds $(6,9)$. The underlying assumption behind such target-predicting methods, which are also widely applied for the study of eukaryotic cell biology $(10,11)$, is that the molecular signatures of bioactive compounds cluster according to the target, irrespective of their structure.

We wanted to probe this assumption and questioned whether or not antibiotics that bind to the same target but belong to different structural classes induced distinguishable molecular signatures. For this purpose, representatives of three classes of DNA gyrase inhibitors, the fluoroquinolones, the aminocoumarins, and the recently discovered cystobactamids, were selected and compared with respect to their metabolome and transcriptome effects on the opportunistic human pathogen Pseudomonas aeruginosa. The Gram-negative, opportunistic pathogen $P$. aeruginosa is a causative agent for nosocomial infections and for infections associated with cystic fibrosis, classified as a priority 1 pathogen by the WHO (12). P. aeruginosa shows substantial resistance to antibiotics by expression of multidrug efflux pumps and establishes infection by specific virulence factors, such as phenazines and rhamnolipids (13). The gyrase Abinding fluoroquinolones, in particular ciprofloxacin, constitute a standard treatment of $P$. aeruginosa infections (14). Subinhibitory concentrations of ciprofloxacin induce phenotypic alterations such as a reduction in swimming and swarming $(14,15)$ but also modulate the expression of hundreds of genes $(14,16,17)$.

Recently, a novel class of gyrase inhibitors, the cystobactamids, were isolated from the soil myxobacterium Cystobacter sp. $(18,19)$. It could be shown in gyrase supercoiling assays and gyrase mutant assays that cystobactamids stabilize the gyrase cleavage complex, indicating that they are type II topoisomerase poisons (18). Cystobactamids engage the gyrase A subunit for target binding as well as the minor groove of the bound DNA through the right-hand side of the molecule (20). Medicinal chemistry efforts lead to cystobactamid analogs like CN-DM-861 and AR351, which showed improved activities against $P$. aeruginosa and other bacterial pathogens compared to those of the natural products $(21,22)$. As a third class, we included the aminocoumarins, which are competitive inhibitors of the ATP-binding site of the gyrase B subunit (23). Crystal structures revealed that the deoxy-sugar of the aminocoumarins overlaps the ATP-binding site and thereby inhibits the catalytic activity of the ATPase $(24,25)$. In contrast to cleavage-complex-stabilizing compounds or gyrase poisons, aminocoumarins do not cause DNA double-strand breaks (25).

While transcriptome effects of ciprofloxacin on $P$. aeruginosa have been reported before $(15-17,26)$, this study represents the first integrative and systematic investigation of the cellular effects associated with gyrase inhibition across three drug classes of gyrase inhibitors.

\section{RESULTS AND DISCUSSION}

Gyrase inhibitors at subinhibitory concentration induce class-specific largescale changes in Pseudomonas aeruginosa PA14 transcriptome and metabolome. In order to identify and compare interclass versus intraclass effects of gyrase inhibitors on the bacterial transcriptome and metabolome, the $P$. aeruginosa model strain PA14 was exposed to the three fluoroquinolones ciprofloxacin, levofloxacin, and lomefloxacin, the two aminocoumarins novobiocin and coumermycin A1, and the two cystobactamids CN-DM-861 and AR351 (Fig. 1a). To avoid that the observed effects simply reflected consequences of altered growth, subinhibitory concentrations were applied 
a

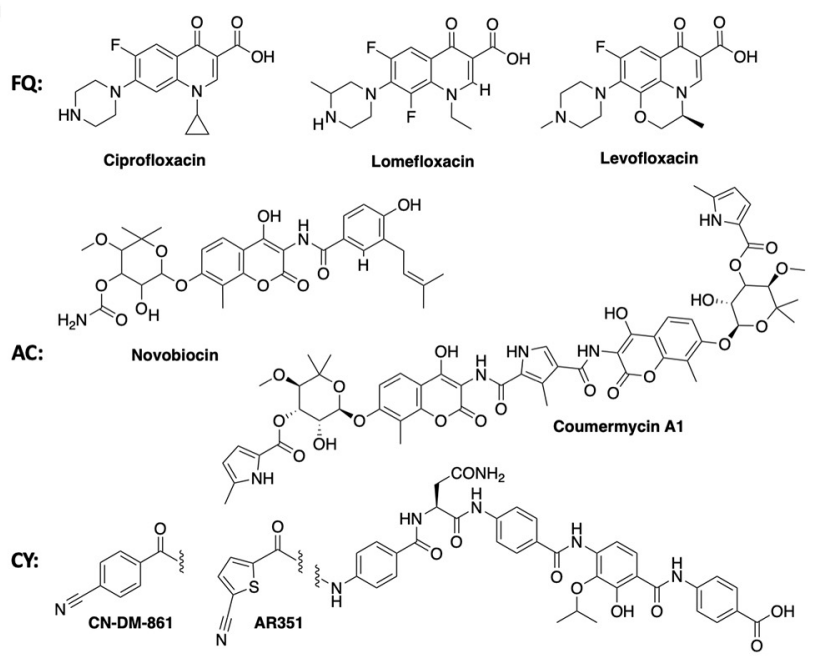

b

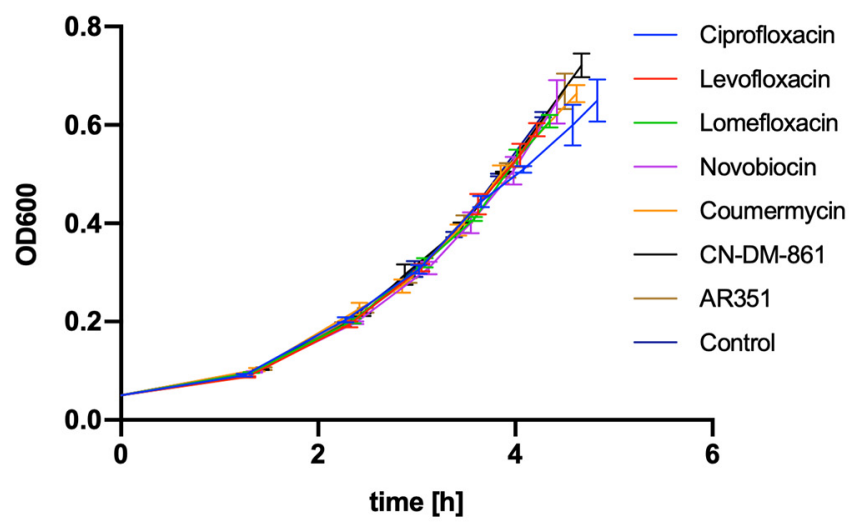

C

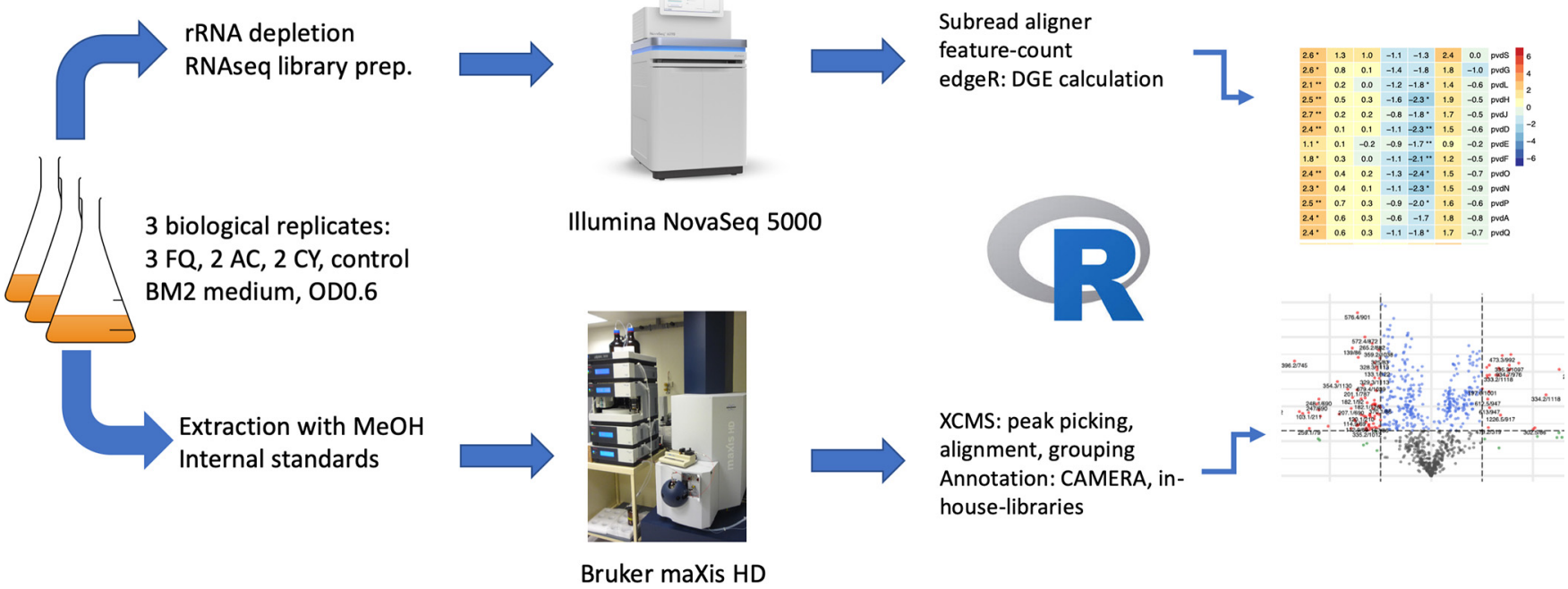

FIG 1 Study design. (a) Chemical structures and names of the antibiotics used in this study. The chemical class (FQ for fluoroquinolones, AC for aminocoumarins, CY for cystobactamids) is indicated to the left. (b) Growth curves of the P. aeruginosa PA14 cultures after treatment with antibiotics at $\mathrm{IC}_{50}$. Curves display the mean of biological triplicates, and the error bars show the standard deviation. (c) Multi-omics workflow used for this study.

that did not impair growth until the target optical density (OD) of 0.6 , corresponding to a mid-exponential phase, was reached. We selected the respective $50 \%$ inhibitory concentration $\left(\mathrm{IC}_{50}\right)$ as the sub-MIC of the compounds (Table 1), as these conditions did not impair growth but allowed to capture the transcriptional and metabolic response to the antibiotic challenge after several hours of exposure. The obtained growth curves were identical, and a minimal retardation of growth for ciprofloxacintreated cultures was nonsignificant (Fig. 1b).

The metabolomes and transcriptomes were detected by untargeted liquid chromatography coupled to (tandem) mass spectrometry (LC-MS/MS) and RNA sequencing, respectively, according to a workflow depicted in Fig. 1c. RNA and metabolites were isolated from the same cultures at the target $O D$ to ensure correspondence of transcriptome and metabolome. Metabolites were extracted using $80 \%$ methanol, separated by reverse-phase chromatography, and analyzed using electrospray ionization-high-resolution time-of-flight mass spectrometry (ESI-QTOF-MS). The extraction protocol was a compromise in order to access a fraction of primary metabolites and also to cover the richness of $P$. aeruginosa's secondary metabolome. The secondary metabolome was of particular interest to us because of the emerging 
TABLE 1 Antibacterial activities and number of regulated transcripts and metabolite features of gyrase inhibitors

\begin{tabular}{|c|c|c|c|c|c|c|c|}
\hline Antibiotic & Class & $\begin{array}{l}\text { MIC } \\
(\mu \mathrm{g} / \mathrm{ml})\end{array}$ & $1 C_{50}(\mu \mathrm{g} / \mathrm{ml})$ & $\begin{array}{l}\text { No. genes } \\
\text { upregulated }^{a}\end{array}$ & $\begin{array}{l}\text { No. genes } \\
\text { downregulated }^{a}\end{array}$ & $\begin{array}{l}\text { No. features } \\
\text { upregulated }{ }^{a}\end{array}$ & $\begin{array}{l}\text { No. features } \\
\text { downregulated }^{a}\end{array}$ \\
\hline Ciprofloxacin & Fluoroquinolone & 0.1 & 0.06 & 343 & 179 & 63 & 87 \\
\hline Levofloxacin & Fluoroquinolone & 0.2 & 0.1 & 172 & 45 & 20 & 77 \\
\hline Novobiocin & Aminocoumarin & 388 & 213 & 277 & 444 & 48 & 176 \\
\hline Coumermycin A1 & Aminocoumarin & 5.3 & 3.8 & 512 & 825 & 123 & 165 \\
\hline CN-DM-861 & Cystobactamid & 3.9 & 1.8 & 163 & 14 & 60 & 10 \\
\hline
\end{tabular}

${ }^{a}$ Differentially expressed genes (fold change $>2$, FDR $<0.05$ ), differential abundance of metabolite features (fold change $>1.5, P<0.05$ ),

understanding that subinhibitory concentrations of antibiotics function as signals to activate bacterial secondary metabolism (27). Data preprocessing led to a peaktable with 836 features. After deisotoping and removal of features that stem from internal standards or the applied gyrase inhibitors, 657 features remained, of which 123 could be annotated (Tables S1 and S3).

After RNA was isolated and purified, the constructed CDNA library was sequenced on a NovaSeq device $(2 \times 50 \mathrm{bp})$, followed by short read-alignment, count estimation, and differential expression analysis.

We first performed a principal-component analysis (PCA) and a hierarchical clustering on both the transcriptome and metabolome data. All scaled and centered features were used for the PCA of the metabolomics data, and centered $\log _{2}$-transformed counts per million (log CPM) data of all 5,979 transcripts were used for the PCA of the transcriptomics data. The PCA score plots of transcripts showed a complete separation of the fluoroquinolones, aminocoumarins, cystobactamids, and the untreated controls for all biological replicates (Fig. 2a). In the PCA score plot of the metabolite features, the classes were also separated, but the $95 \%$ confidence ellipses were more spread out, and the cystobactamids partially overlapped the fluoroquinolones. For hierarchical cluster analysis, all pairwise Euclidean distances using the normalized and scaled metabolite features (Fig. 2b, left) and log CPM values of the transcripts (Fig. 2b, right) were calculated. For the RNA-Seq data, three main clusters were formed. The aminocoumarins were grouped in one cluster, with the biological replicates in closest proximity that had the largest distance to all others. The fluoroquinolones spanned a second cluster, with levofloxacin replicates in one subcluster and ciprofloxacin and lomefloxacin in another. The third cluster was split into a cystobactamid subcluster and into a subcluster with the untreated control replicates, indicating that cystobactamids exerted the smallest effect on PA14 transcriptome and showed the highest similarities to the untreated controls. The clustering of the metabolomics data gave a similar result, with the clearest separation of the aminocoumarin cluster from the others. Also, the fluoroquinolones spanned a distinct subcluster. Cystobactamids split into two subclusters, with AR351 replicates in closer proximity to the fluoroquinolones, whereas the CN-DM-861 cluster was grouped closer to the untreated control. Overall, fluoroquinolones, aminocoumarins, and cystobactamids have distinct effects on the transcriptome and metabolome of PA14 and can be distinguished according to their class; the gyrase B-binding aminocoumarins were distant from the gyrase A-binding fluoroquinolones and cystobactamids. The differences cannot be traced back to a technical variance of the method, because both biological replicates of a given compound as well as different congeners of a compound class (i.e., ciprofloxacin, levofloxacin, and lomefloxacin) were more similar to each other than to members of other classes. While a complete separation was achieved for the transcriptome data, metabolome data had a broader spread. This can be explained with the higher experimental variability of the metabolomics extraction protocol. In addition, the expression values of all 5,979 transcripts were assessed, whereas only the midpolar fraction with an emphasis on secondary metabolites of the PA14 metabolome was sampled, thus obscuring a potential class separation by nonrepresented metabolites undetected. 
a

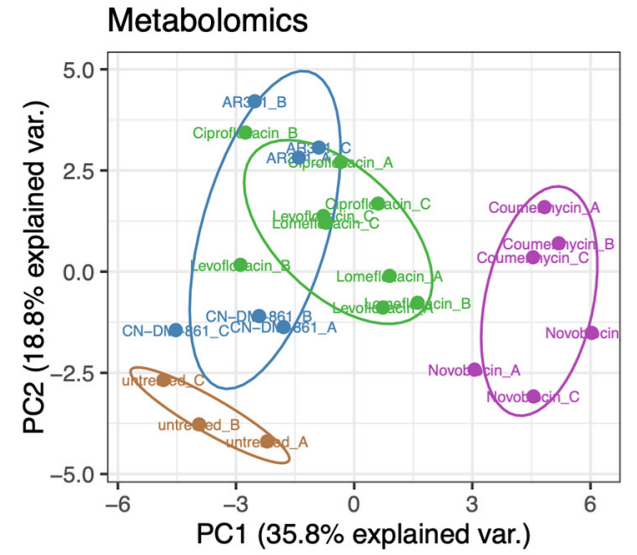

b

\section{c}
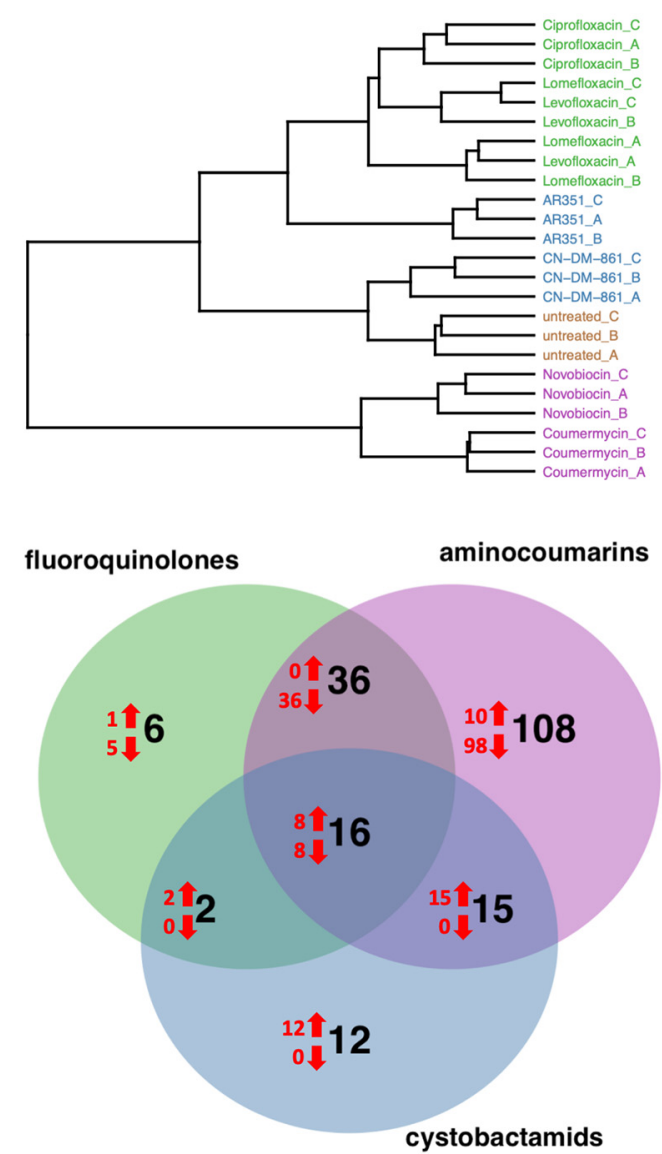
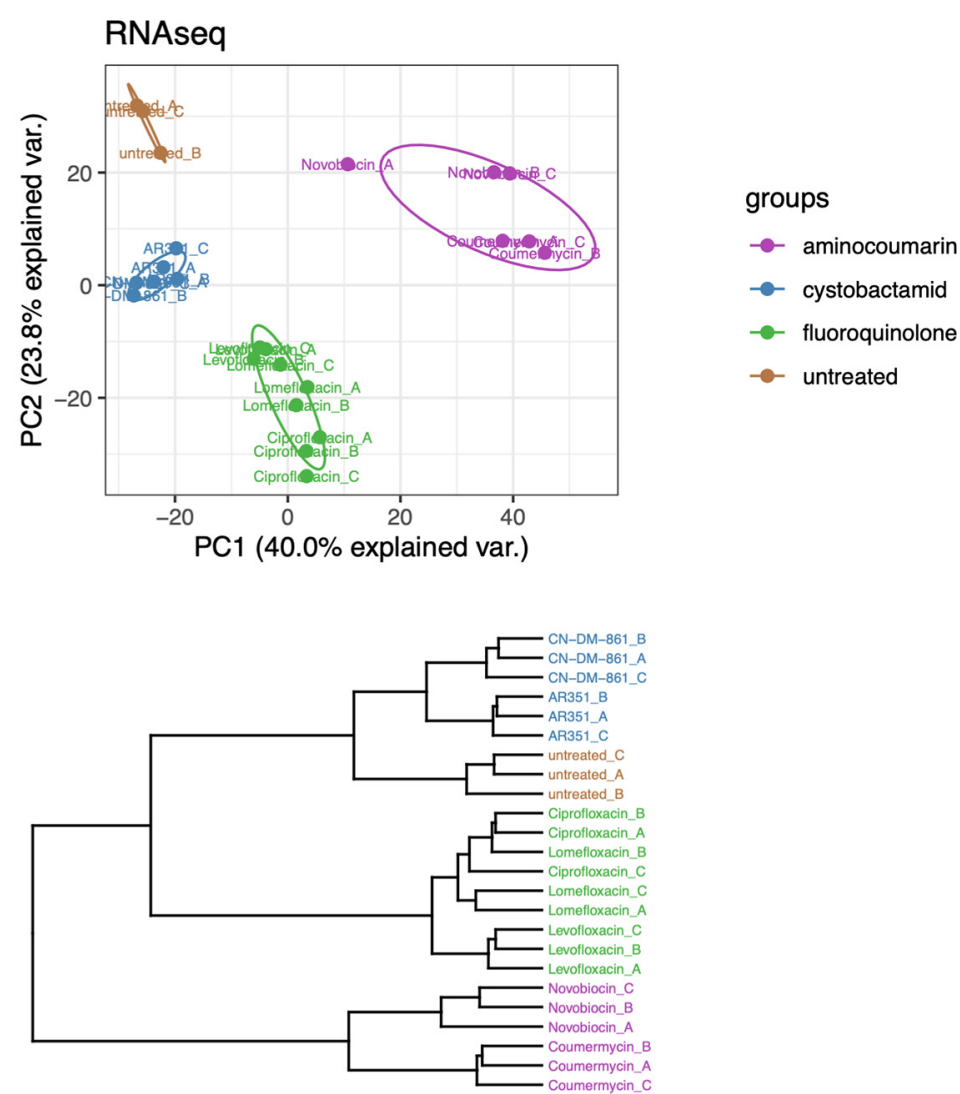

fluoroquinolones aminocoumarins

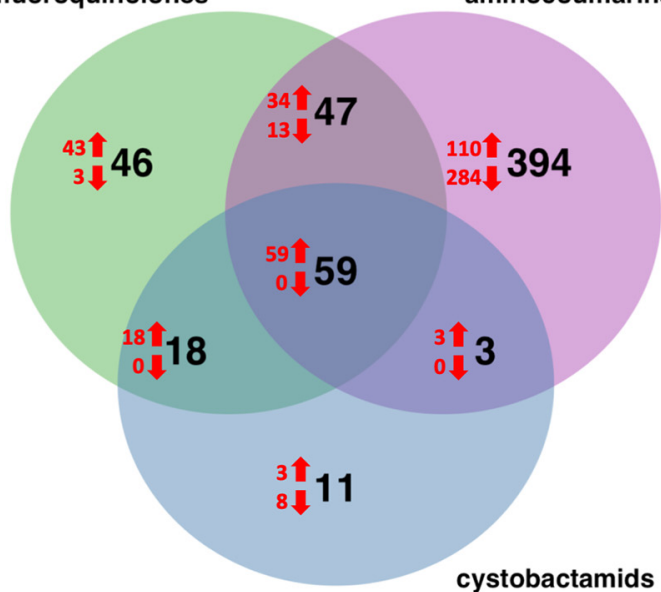

FIG 2 Global outcome of transcriptome and metabolome analysis. (a) Principal-component analysis (PCA) scores plots. Left, metabolome, calculated from all scaled and centered metabolite features; right: transcriptome, centered log CPM expression data of all genes. (b) Hierarchical clustering using Ward's linkage criterion. Left, metabolome, Euclidean distances calculated from all normalized and scaled metabolic features; right, transcriptome, Euclidean distance calculated from log CPM expression values. (c) Venn diagram displaying the numbers of differentially abundant metabolic features and transcripts for each class of gyrase inhibitors compared to untreated controls (total numbers in black, numbers in red show up-/downregulated features or genes). Left, differentially abundant metabolic features ( $\log _{2}$ fold change [FC] $>\log _{2}[1.5]$ or $\log _{2}$ FC $<-\log _{2}[1.5]$ and $P<0.05$ ); right, differentially expressed genes $\left(\log _{2} \mathrm{FC}>1\right.$ or $\log _{2} \mathrm{FC}<-1$ and $\left.\mathrm{FDR}<0.05\right)$.

To pinpoint the transcripts and metabolites that contribute to class separation, their relative abundances, expressed as fold changes in comparison to those of the untreated controls, and the associated $P$ values were calculated for all analytes. Cutoffs for fold change of 2 for the transcripts and of 1.5 for the metabolite features were applied. The number of transcripts and metabolite features that are regulated by all members of one inhibitor class are shown in Table 2, and those that are jointly 
TABLE 2 Number of regulated transcripts and metabolite features shared by all members of a gyrase inhibitor class

\begin{tabular}{lllll}
\hline Class & $\begin{array}{l}\text { No. genes } \\
\text { upregulated }^{a}\end{array}$ & $\begin{array}{l}\text { No. genes } \\
\text { downregulated }^{a}\end{array}$ & $\begin{array}{l}\text { No. features } \\
\text { upregulated }^{a}\end{array}$ & $\begin{array}{l}\text { No. features } \\
\text { downregulated }^{a}\end{array}$ \\
\hline Fluoroquinolones & 154 & 16 & 11 & 49 \\
Aminocoumarins & 206 & 297 & 33 & 142 \\
Cystobactamids & 83 & 8 & 37 & 8 \\
\hline
\end{tabular}

${ }^{a}$ Differentially expressed genes (fold change $>2, F D R<0.05$ ), differential abundance of metabolite features (fold change $>1.5, P<0.05)$.

regulated by all members of two or all three classes are depicted in Venn diagrams (Fig. 2c). The degree of regulation found for transcriptome and that found for metabolome correlate well. The numbers of significantly regulated transcripts and differentially abundant metabolites differed strikingly between the antibiotic classes (Tables 1 and 2 and Tables S2 and S3): whereas cystobactamids affected the smallest number of transcripts and metabolic features, the aminocoumarins induced differential effects in the highest number of genes and metabolic features. Particularly regarding downregulation of transcripts and feature levels, the aminocoumarins had by far the most pronounced effect. The fluoroquinolones in comparison also induced upregulation of 154 genes (intersection of the three fluoroquinolones used) but had fewer downregulating effects (Tables 1 and 2). Aminocoumarins also had the highest number of differentially regulated transcripts and metabolic features that are not shared with the two other classes. The Venn diagrams in Fig. $2 c$ show the intersections of the transcripts and metabolic features that are jointly affected by two or three classes of gyrase inhibitors. The pool of transcripts and metabolic features used to construct the Venn diagrams was created by taking the intraclass intersections of regulated transcripts and differentially abundant metabolic features (i.e., regulated transcripts/features shared by all compounds of a class). Overall, the numbers of jointly regulated transcripts and metabolite features (59 and 21, respectively) are far smaller than the numbers of analytes affected by only one (up to 394 transcripts and up to 108 metabolite features) or two classes (up to 47 transcripts and up to 36 metabolite features). Thus, a striking result of this summary analysis is that the three classes of gyrase inhibitors have widely differing (rather than similar) imprints on PA14 transcriptome and metabolome with respect to the cutoff criteria. In the following section, we go through the most striking changes that are caused by gyrase inhibitor perturbation.

Gyrase inhibitors of all classes induce SOS responses. Gyrase poisoning and the resulting DNA double-strand breaks according to previous studies (28) induce the SOS response, which is characterized by RecA-mediated LexA-induced gene expression. Because the LexA regulon of $P$. aeruginosa was first described for PAO1 (16), the PAO1 locus IDs were mapped to the PA14 genome using the BACTOME annotator (29). All gyrase inhibitors investigated in this study induced upregulation of the LexA regulon, albeit to different extents (Fig. 3a). The strongest induction was found for ciprofloxacin, followed by the other fluoroquinolones, whereas the cystobactamids had weaker effects. Surprisingly, the LexA regulon was also induced by the aminocoumarins, although they do not directly cause DNA double-strand breaks. A possible explanation is that DNA damage is caused not only by gyrase A poisoning but also through the action of reactive oxygen species (ROS). In fact, Collins and coworkers have shown that bactericidal antibiotics induce hydroxyl radical formation through an oxidative damage pathway, which then triggers RecA and the DNA damage response (30). The presence of ROS triggers cellular protective responses to oxidative modifications of proteins (e.g., carbonylation) (31). We indeed observed an upregulation of chaperones and heat shock proteins indicating protein damage (Fig. 3a). Thus, we speculate that the aminocoumarins elicit a strong protective response to ROS via upregulation of heat shock proteins and chaperones and a subsequent RecA-mediated SOS induction without causing direct DNA damage. 
a
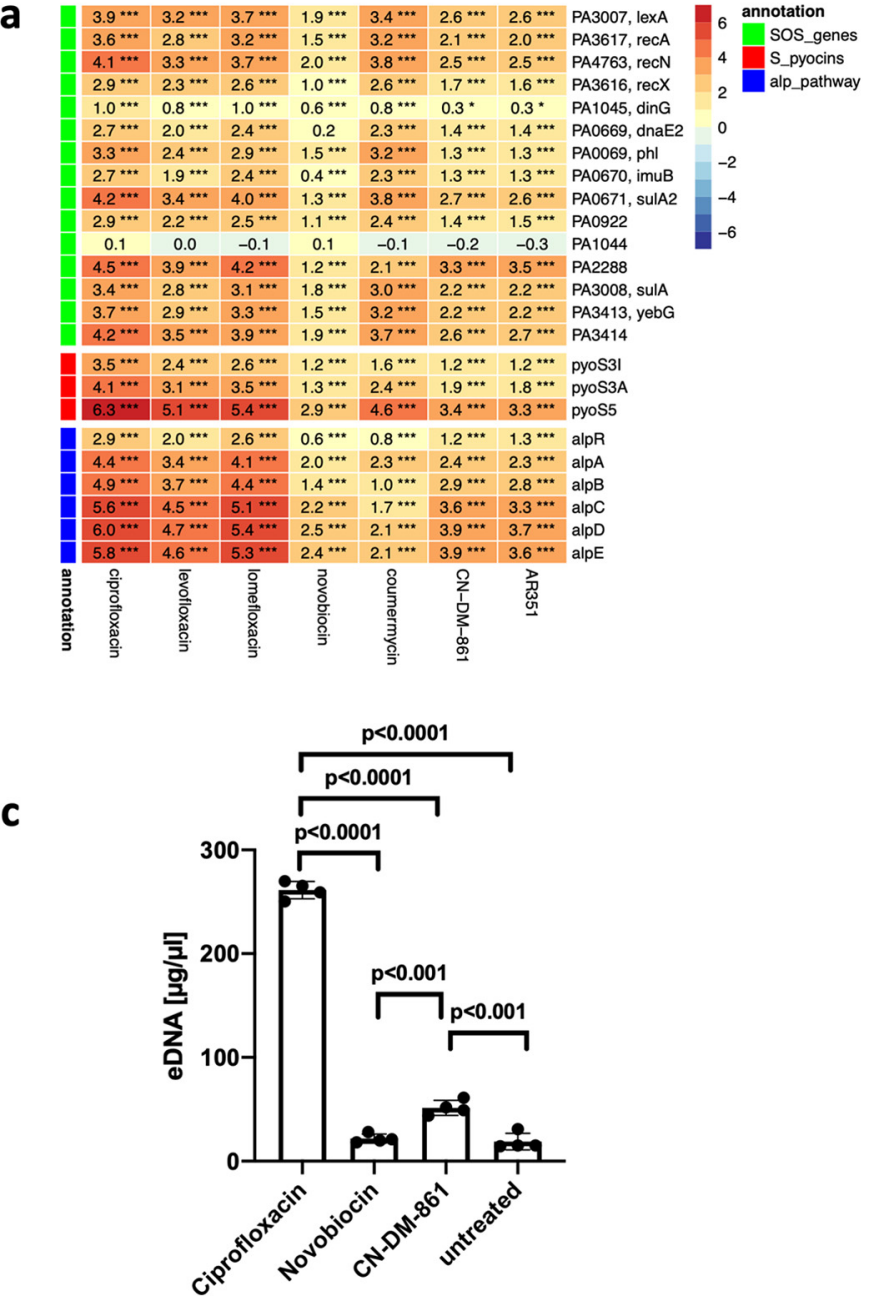

b

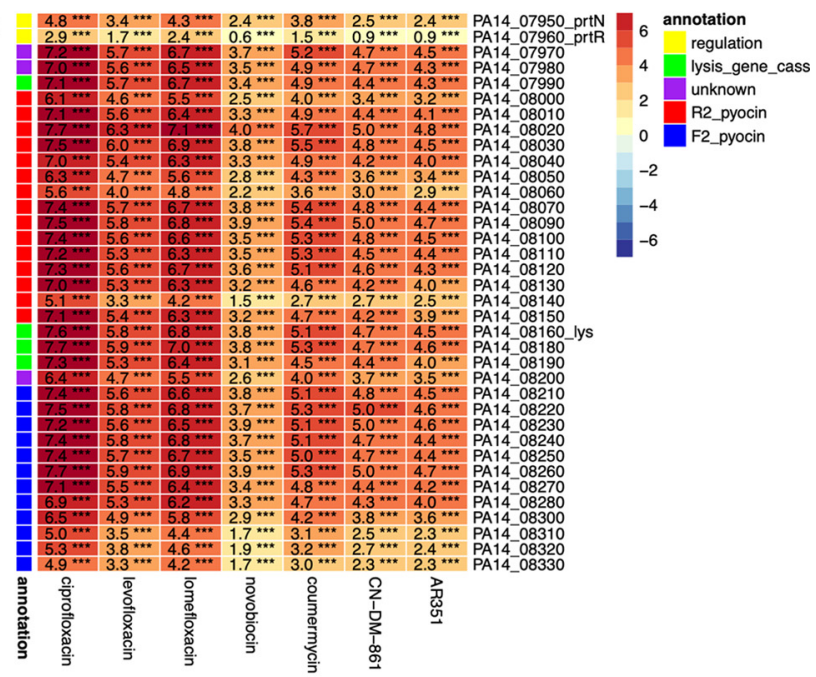

d

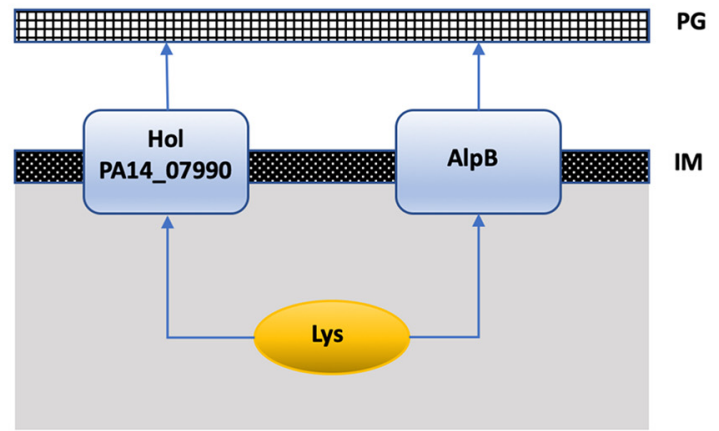

FIG 3 (a) Heatmap of differentially expressed genes of the SOS regulon, as defined by Cirz et al. (16), heatshock and chaperone genes, S-pyocins, and the Alp cell lysis pathway. PAO1 locus tags were mapped to PA14 locus tags where necessary. Numbers display the $\log _{2}$ fold change (log $\mathrm{FC}$ ) in comparison to the untreated control, which is also translated into the color scale. Number of asterisks denotes FDR thresholds: ${ }^{* * *}, 0.001 ;{ }^{* *}, 0.01{ }^{*}, 0.05$. (b) Heatmap of differentially expressed genes of the prophage/RF-pyocin region. (c) Quantification of eDNA from planktonic cultures of PA14 at OD of 0.6, treated with ciprofloxacin $(n=4)$, novobiocin $(n=4), C N-D M-861(n=4)$, and buffer control $(n=4)$. The $P$ values were determined by ordinary one-way analysis of variance (ANOVA) with Bonferroni's multiple-comparison test $(F=1,047.0 ; d f=15)$. Error bars show standard deviation. (d) Scheme showing endolysinmediated autolysis in $P$. aeruginosa adapted from reference 13 .

Strong upregulation of pyocin regions and Alp pathway and detection of eDNA verify self-cell lysis in response to gyrase inhibitor treatment. The most pronounced upregulation of gene expression as a consequence of gyrase inhibitor treatment could be mapped to the so-called prophage R/F-pyocin region of PA14. The effect was strongest for fluoroquinolones; in particular, ciprofloxacin induced up to 212 -fold changes in transcript abundance ( $\log _{2}$ fold change of 7.73) (Fig. 3b).

The upregulation of the R/F-pyocin region within the PAO1 genome was described in response to the treatment with hydrogen peroxide as a consequence of oxidative stress (32). It was also observed as a consequence of ciprofloxacin treatment of $P$. aeruginosa strains PAO1 and PAK $(26,33)$. We mapped the PAO1 locus IDs to orthologs in the PA14 genome and found exactly the same region to be affected by each gyrase inhibitor used in this study.

Pyocins are protein toxins that are used in interspecies competitions against the same or closely related strains. P. aeruginosa produces three types of pyocins: The Stype pyocin is a colicin-like protein, and the R- and F-type pyocins are derived from phage tails and are evolutionary specialized as bacteriocins (13). It has been shown that clinical isolates of $P$. aeruginosa produce distinct ranges of pyocins, which 
influence biofilm formation and the strain composition in the cystic fibrosis (CF) lung (34) and thus clinical outcome. The R/F-pyocin region is regulated by $p r t N$, a positive regulator of pyocin production under the control of the prtR repressor. RecA causes the autocleavage of PrtR, leading to the expression of $p r t N$ and upregulation of expression of pyocins (35), and thereby links the SOS response directly to pyocin induction.

In fact, there is a qualitative correlation between the strength of SOS response and pyocin production by the different gyrase inhibitors in this study. Novobiocin treatment causes the weakest SOS induction and also the weakest pyocin induction, whereas ciprofloxacin is the strongest inducer for both processes.

Within the R/F-pyocin region, a putative holin protein (PA14_07990) and the endolysin Lys (PA14_08160) are encoded. According to Turnbull et al., Lys crosses the inner membrane via holin proteins and degrades the peptidoglycan cell wall, which eventually leads to cell lysis (36) (Fig. 3d). Self-lysis is also induced by the holin protein AlpB through the Alp pathway (37). Cleavage of the regulator AlpR leads to derepression of the alpA gene, which encodes a positive regulator that activates the expression of the alpBCDE lysis cassette (37). The Alp pathway contributes to the lethality for the individual cell, although the whole population might benefit by release of eDNA and biofilm formation.

We found that both lys and the Alp pathway were consistently upregulated, again with the fluoroquinolones eliciting the strongest effects, followed by the cystobactamids, whereas the aminocoumarins induced the smallest fold changes.

The autolysis of a fraction of the $P$. aeruginosa population should lead to the release of DNA into the medium. We therefore investigated whether the induction of the RFpyocin region correlated with the amount of released extracellular DNA (eDNA). For this purpose, eDNA was quantified following a protocol by Allesen-Holm et al. using the same cultivation conditions as for the omics experiments (38), with one representative of each gyrase inhibitor class (Fig. 3c). For ciprofloxacin, the eDNA concentration of $261.28 \mu \mathrm{g} / \mu \mathrm{l}$ released to the medium exceeded the control value of $18.86 \mu \mathrm{g} / \mu \mathrm{l}$ by $\operatorname{far}(P<0.0001)$. Also, the treatment with the cystobactamid CN-DM-861 led to a significant increase of eDNA $(51.48 \mu \mathrm{g} / \mu \mathrm{l}, P<0.001)$. In contrast, novobiocin induced no statistically significant difference compared to the control. We also tested for association between the expression of the endolysin lys in CPM and the amount of eDNA detected in $\mu \mathrm{g} / \mu \mathrm{l}$ and obtained a Spearman rank correlation coefficient $\rho$ of $1(P=0.083)$, indicating a strong positive correlation (Fig. S1). This is a clear hint that indeed the postulated mechanism of endolysin/holin upregulation leads to degradation of the peptidoglycan layer, destabilization of the cell wall, and finally autolysis of the bacterial cell. Self-lysis may contribute substantially to the efficacy of gyrase inhibitors, irrespective of their class.

Aminocoumarins induce a strong downregulation of virulence factors. The pathogenicity of $P$. aeruginosa is strongly mediated by low-molecular-weight virulence factors, including the phenazines, the rhamnolipids, and quorum-sensing mediators (39). The phenazines are redox-active heterocycles that contribute to pathogenesis by subjecting host cells to harmful oxidative stress (40). Two almost identical operons (phz1 and phz2) code for the phenazine biosynthesis pathway, which produces phenzine-1-carboxylic acid as the precursor for all other phenazines, that is further converted to pyocyanin by PhzM and PhzS (41).

We observed a strong downregulation of both $p h z 1$ and phz2 operons for aminocoumarin-treated samples (Fig. 4a). Correspondingly, the concentrations of phenazine metabolites like phenazine-1-carboxylic acid and pyocyanin were strongly decreased (Fig. 4b and Table S2). Smaller or nonsignificant downregulating effects were exerted by fluoroquinolones and cystobactamids, respectively.

The rhamnolipids, glycolipids with surfactant-like properties, span another class of important virulence factors, because they enable $P$. aeruginosa to eliminate leucocytes and evade the most important immune mechanisms in the CF lung (13). The transcripts $r h / A$, $r h / B$, and $r h / C$, which encode key enzymes that attach the fatty acids to rhamnose units, 
a

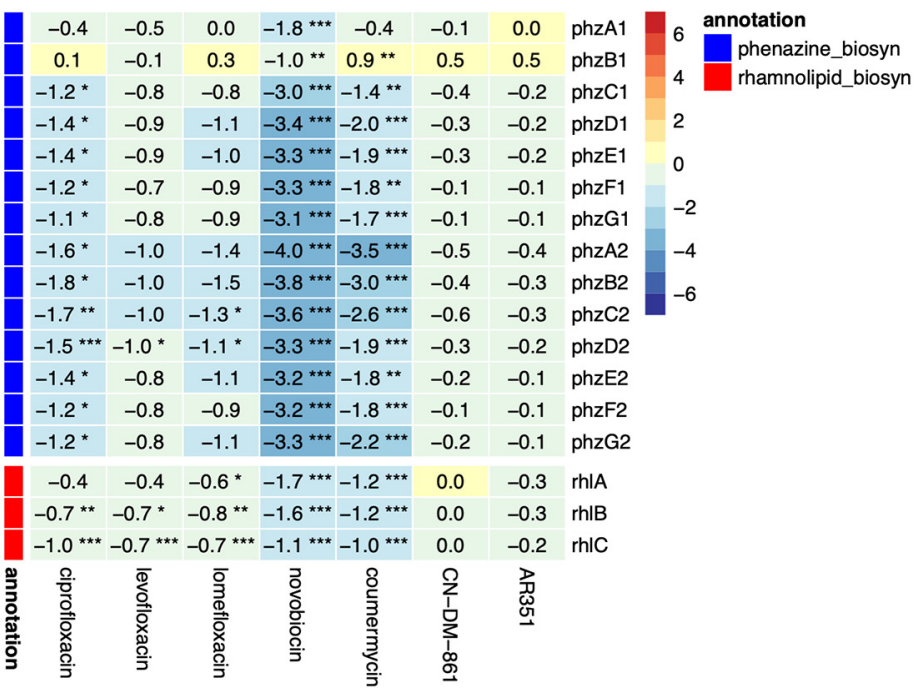

b
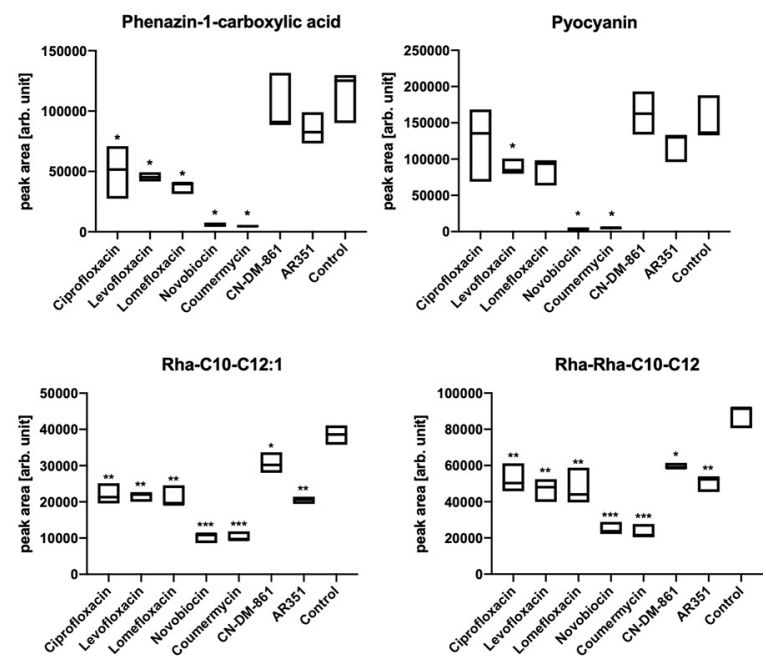

FIG 4 (a) Heatmap displaying $\log _{2} F C$ values and FDR values for two phz operons coding for phenazine biosynthesis (blue) and the genes rhlABC coding for rhamnolipid synthesis (red). Number of asterisks denotes FDR thresholds: ${ }^{* * *}, 0.001 ;{ }^{* *}, 0.01 ;{ }^{*}, 0.05$. (b) Box plots displaying the distributions of the abundance of representative metabolites of the phenazine and rhamnolipid class. Asterisks denote $P$ values for comparison to the untreated controls $\left(* * *, 0.001 ;{ }^{* *}, 0.01 ;{ }^{*}, 0.05\right)$.

were strongly downregulated upon aminocoumarin treatment (Fig. 4a). In line with this, decreased rhamnolipid concentrations were found in the metabolome (Fig. 4b). Thus, a differentiating property of the aminocoumarins, reflected on the transcriptome and metabolome level, lies in their strong downregulating effect on virulence factors.

Cystobactamids induce a unique downregulation of a glucose catabolism pathway. The only consistently downregulated pathway we found for the cystobactamids concerns two operons that were not fully annotated in the PA14 genome but could be matched to the 2-ketogluconate utilization operon of PAO1 (42) and the gad (gluconate dehydrogenase) operon (43) (Fig. 5).

$P$. aeruginosa metabolizes glucose exclusively via the Entner-Doudoroff pathway (44). Glucose enters the periplasm via an OprB porin and is then metabolized via three parallel routes that all converge on 6-phosphogluconate (45) (Fig. 5a). One of the routes comprises the 2 -fold oxidation of glucose to gluconate and further to 2-ketogluconate in the periplasm, the transport of 2-ketogluconate to the cytoplasm, its phosphorylation by KguK to 2-keto-6-phophogluconate, and a final reduction to 6phosphogluconate, which enters the Entner-Doudoroff pathway. This branch was selectively downregulated by cystobactamid treatment, whereas fluoroquinolones or aminocoumarins induced weak or nonsignificant effects. However, it is unclear whether addressing this arm of primary metabolism has an impact on the overall glucose metabolism of $P$. aeruginosa PA14. For $P$. aeruginosa PAO1, it has recently been shown by flux analysis approaches that approximately $90 \%$ of its glucose is oxidized into gluconate via the periplasmic route (46). Strikingly, the porin OprB, which mediates glucose uptake across the outer membrane, was strongly downregulated for the fluoroquinolones (2- to 4-fold), less (1.6- to 2.6-fold) for the aminocoumarins, and only 1.3- to 1.7-fold for the cystobactamids. In the case of the cystobactamids, glucose utilization was additionally diminished by downregulating enzymes of the 2-ketogluconate utilization operon, whereas for the other two classes, this effect was achieved by regulating OprB alone.

General discussion. Efforts to use untargeted metabolomics for mode-of-action studies of bioactive compounds rely on the assumption that molecules addressing a given target induce similar molecular signatures. The current study puts a note of caution on this assumption by demonstrating that there are clear differences depending on the drug class. Unsurprisingly, these were pronounced when different subunits with distinct functions, i.e., gyrase A versus gyrase B, were targeted. But even 
a

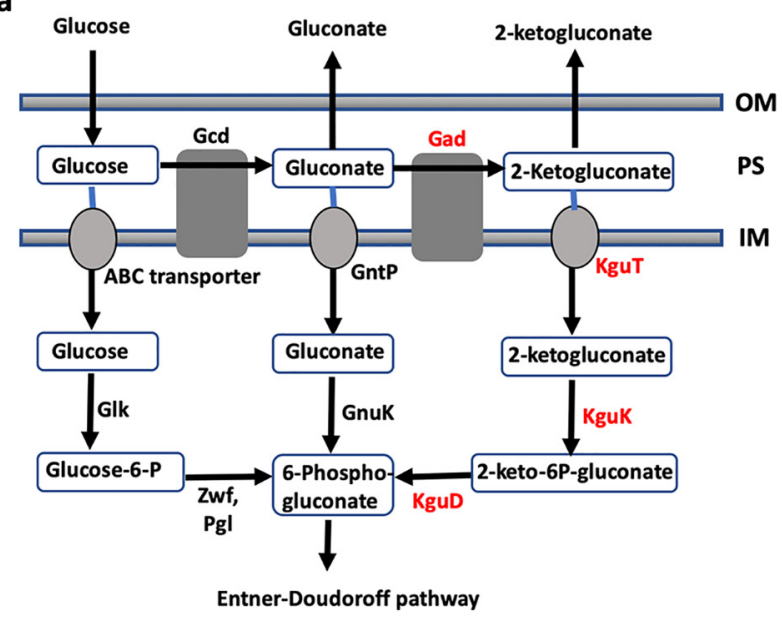

b

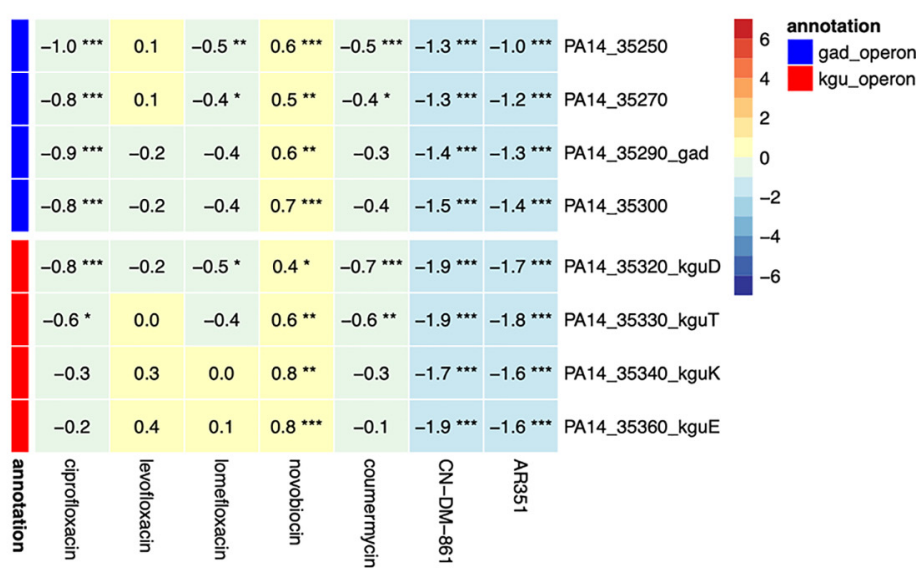

FIG 5 (a) Scheme of glucose utilization pathways in Pseudomonas subspecies, adapted from references 73 and 74. OM, outer membrane; PS, periplasmic space; IM, inner membrane; Gcd, glucose dehydrogenase; Gad, gluconate dehydrogenase; GntP, gluconate transporter; KguT, 2-ketogluconate transporter; Glk, glucokinase; GnuK, gluconate kinase; KguK, 2-ketogluconate kinase; Zwf, glucose-6-phosphate dehydrogenase; Pgl, 6-phosphogluconolactonase; KguD, 2-keto-6-phosphogluconate reductase. Downregulated enzymes are shown in red. (b) Heatmap displaying $\log _{2}$ FC values and FDR values for the 2ketogluconate (kgu) utilization operon (42) and the gad operon coding for the enzymes involved in transport and conversion of 2-ketogluconate into 6phosphogluconate, which is then funneled into the Entner-Doudoroff pathway as shown in panel a (43). Number of stars denote FDR thresholds: ${ }^{* * *}, 0.001 ;$ $* *, 0.01 ;{ }^{*}, 0.05$.

compounds addressing proximal binding sites on the same subunit like fluoroquinolones and cystobactamids could be differentiated by the transcriptomic, and to a lesser extend metabolomic, changes they induce in Pseudomonas aeruginosa PA14. A part of the discrimination can be attributed to differing sizes of effects going to the same direction, with some treatments falling above cutoff criteria and others below, whereas strong and significant effects going into opposite directions are rare. Thus, the cellular effects of different gyrase inhibitors are not fundamentally different. However, the findings have implications for mode-of-action assignments based on correlation methods. Ideally, more than one drug class for given targets or mechanisms is required to properly validate an inter- versus intratarget spread of signatures.

In this gyrase study, the aminocoumarins stand out by exerting the broadest effect and inducing the highest number of differentially regulated genes/metabolite features. We noted their strong downregulation of phenazines and rhamnolipids, important virulence factors of $P$. aeruginosa. The pronounced anti-virulence activity even at subinhibitory concentrations suggests a potential role for aminocoumarins as pathogenicity blockers of $P$. aeruginosa infections beyond that of a classical antibiotic, in particular when combined with compounds that exert synergism in PA14 like chlorhexidine or colistin (47).

Exposure to aminocoumarins also led to a surprisingly strong SOS response, which has been a hallmark of gyrase A poisoning. This observation contrasts a study in Staphylococcus aureus that describes an inhibiting effect of novobiocin at higher, not subinhibitory, concentrations on the ciprofloxacin-induced SOS response (48). On the other hand, the activation of the SOS response has been observed in Escherichia coli (49) and in Bacillus subtilis (50) following aminocoumarin treatment. We speculate that reactive oxygen species, indicated by an upregulation of stress response genes, are mediating DNA damage and subsequent induction of the SOS response.

The fact that aminocoumarins induce a much higher number of differentially expressed genes could be explained by the different inhibition mechanism of the gyrase. The DNA gyrase introduces negative supercoils in an ATP-dependent manner to maintain the DNA in an underwound state (51). By blocking the ATP-binding site, aminocoumarins cause partial relaxation toward a relaxed state (51). It has been shown that gene expression is directly influenced by the degree of supercoiling, with some genes showing enhanced expression when DNA is relaxed, while others are more 
highly expressed when negative supercoils are introduced $(51,52)$. Several studies demonstrate an effect of supercoiling on transcription initiation and detect bacterial genes that exhibit changes in expression that correlate with changes in DNA supercoiling (51-53). Increased amounts of exposed single-stranded DNA (ssDNA) as a consequence of negative supercoiling induced by the aminocoumarins provide an alternative explanation for the triggering of the SOS response (54). The binding of RecA to ssDNA leads to an increased proteolytic cleavage of LexA by RecA, thereby inducing expression of the LexA regulon (55).

A potential contributor to the observed differences between classes is off-target effects. Network-based approaches led to the finding that an average number of target proteins per drug of 6.3 can be calculated (56). Chemical proteomics approaches have revealed that it is also not uncommon to find multiple targets for antibiotics (57). For instance, vancomycin was found to inhibit the major staphylococcal autolysin Atl in addition to the primary mechanism of action exerted by binding to the dipeptide termini of peptidoglycan (58). Novobiocin inhibited the eukaryotic heat shock protein HSP90 by interacting with an ATPase binding domain (59), and an inhibition of bacterial ATPases might in principle contribute to the unique aminocoumarin signature observed here. The risk of hitting off-targets was deemed to be particularly high for novobiocin, because the applied $\mathrm{IC}_{50}$ was substantially higher than those of the other antibiotics. However, the number of regulated genes and features was actually 2-fold lower than that for coumermycin, which was applied at an $\mathrm{IC}_{50}$ in the low $\mu \mathrm{M}$ range, comparable to the other substances.

A striking finding of our study concerns the strong induction of pyocin production, ultimately leading to auto cell lysis, as demonstrated by the detection of eDNA. Pyocin upregulation in $P$. aeruginosa has been detected before, after triggering by hydrogen peroxide, ciprofloxacin, or genotoxic agents such as mitomycin $C(32,36-38,60,61)$. We show that the level of pyocin induction depends very much on the inhibitor class, with the fluoroquinolones inducing the strongest effects, and that the release of eDNA correlates directly with the endolysin expression. Evolutionarily, this altruistic suicide (36) of a bacterial subpopulation only makes sense by providing a survival advantage for the whole population, e.g., by releasing eDNA and enhancing biofilm formation (35). In fact, biofilm formation has been shown to drastically impair the efficacy of antibiotics such as fluoroquinolones (62). While this caveat concerns all compounds studied here, it remains to be probed whether the less pronounced pyocin induction of cystobactamids may translate to advantages in terms of biofilm formation.

Conclusions. Through a combination of transcriptomics and metabolomics, we demonstrated that the molecular signatures induced by three classes of gyrase inhibitors differ from each other. The findings enhance our understanding on the mode of actions of these important current (fluoroquinolones, aminocoumarins) and potential future (cystobactamids) antibiotics. They also suggest that signature correlation methods for target identification should consider intra-target-class variances. The differential effect of the three gyrase inhibitor classes is likely being caused by a combination of on- and off-target effects. To tease these effects apart, we plan to use resistant mutants of PA14 with strongly reduced target affinity. Another logical expansion of the approach to further gyrase A and B inhibitor classes, or to other prominent antibiotic targets (e.g., protein translation, cell wall synthesis), is also subject to future studies.

\section{MATERIALS AND METHODS}

Reagents. Cystobactamids CN-DM-861 and AR351 were synthesized in the lab of the corresponding author according to reference 21. Ciprofloxacin was purchased from AppliChem. Coumermycin A1, levofloxacin, lomefloxacin $\mathrm{HCl}$, novobiocin sodium salt, trimethoprim, nortriptyline $\mathrm{HCl}$, caffeine, $\left(\mathrm{NH}_{4}\right)_{2} \mathrm{SO}_{4}$, and $\mathrm{Fe}(\mathrm{II}) \mathrm{SO}_{4} 7 \times \mathrm{H}_{2} \mathrm{O}$ were obtained from Sigma-Aldrich. Glipizide was purchased from ACROS Organics, and (S)-naproxen was purchased from Cayman Chemical Company. $\mathrm{D}(+)$-Glucose monohydrate and $\mathrm{K}_{2} \mathrm{HPO}_{4}$ were obtained from Merck Millipore, and $\mathrm{MgSO}_{4} 7 \times \mathrm{H}_{2} \mathrm{O}$ and casamino acid were obtained from Roth. Acetonitrile ultra LC/MS grade, water ultra LC/MS grade, and methanol ultra LC/MS grade were obtained from Fisher Scientific. 
Bacteria. The Pseudomonas aeruginosa PA14 strain was obtained from the DSMZ (DSM 19882).

MIC and IC ${ }_{50}$ determination via microdilution method. MIC values were determined following the microdilution method but using BM2 medium instead of Mueller-Hinton broth. A starting OD of 0.1 of Pseudomonas aeruginosa PA14 was used as inoculum in BM2 medium. Cultivation at $37^{\circ} \mathrm{C}$ was carried out in microtiter plates.

Compounds were serially diluted in 96-well microtiter plates in triplicate. After incubation of the plates for $18 \mathrm{~h}$ at $37^{\circ} \mathrm{C}$, the absorbance at $600 \mathrm{~nm}$ was measured to determine the MIC value and the $\mathrm{IC}_{50}$ value. We used a Gompertz function to fit the data in $\mathrm{R}$ and determine the $\mathrm{IC}_{50}$ and MIC according to Lambert and Pearson (63).

Bacterial culture and addition of gyrase inhibitors. For profiling of the effects of gyrase inhibitors on the Pseudomonas aeruginosa PA14 metabolome and transcriptome, planktonic bacteria were cultivated to mid-exponential phase $\left(\mathrm{OD}_{600}=0.6\right)$ in the presence of subinhibitory concentration of the antibiotic.

A preculture was prepared by inoculation of $20 \mathrm{ml} \mathrm{BM2}$ medium $\left[2 \mathrm{mM}\left(\mathrm{NH}_{4}\right)_{2} \mathrm{SO}_{4}, 40 \mathrm{mM} \mathrm{K}_{2} \mathrm{HPO}_{4}\right.$, $22 \mathrm{mM} \mathrm{KH}_{2} \mathrm{PO}_{4}, 2 \mathrm{mM} \mathrm{MgSO}_{4}, 10 \mu \mathrm{M} \mathrm{FeSO}_{4}, 0.4 \%$ (wt/vol) glucose, $0.01 \%$ (wt/vol) Casamino Acids] with Pseudomonas aeruginosa PA14 from the glycerol stock. The preculture was shaken $(150 \mathrm{rpm})$ overnight at $37^{\circ} \mathrm{C}$. The 1:10 diluted preculture was then used to calculate the precise dilution to inoculate a $20 \mathrm{ml}$ $\mathrm{BM} 2$ culture at an $\mathrm{OD}_{600}$ of 0.05 . Immediately after adjusting the starting $\mathrm{OD}$, the amount of gyrase inhibitor that resulted in the concentration corresponding to the $\mathrm{IC}_{50}$ that was determined via the broth microdilution method was added to the culture (see Table 1).

The compounds were added from a dimethyl sulfoxide (DMSO) stock solution, apart from lomefloxacin and novobiocin that were added from an $\mathrm{H}_{2} \mathrm{O}$ stock solution. For each flask it was ensured that the same amount of DMSO was added, so in the case of lomefloxacin and novobiocin, $10.1 \mu$ l of DMSO was additionally added, and for the untreated control we used a mock treatment of $10.1 \mu \mathrm{l}$ DMSO as well. For each gyrase inhibitor that was tested and for the control, three independent $20 \mathrm{ml}$ main culture flasks were prepared and incubated at $37^{\circ} \mathrm{C}$ and $150 \mathrm{rpm}$ until an OD of 0.6 was reached. The OD was monitored regularly and growth curves were recorded. At the target $\mathrm{OD}$ of $0.6,2 \mathrm{ml}$ of culture was transferred to an Eppendorf tube and centrifuged for $5 \mathrm{~min}\left(9,000 \times 9,4^{\circ} \mathrm{C}\right)$ for metabolomics measurements. The supernatant was discarded, and the pellet was washed with $0.9 \% \mathrm{NaCl}$ and frozen with liquid nitrogen. For RNA-Seq measurements of the same sample, $0.75 \mathrm{ml}$ from the same culture (at OD of 0.6) was added to an Eppendorf tube with $0.75 \mathrm{ml}$ RNAprotect (Qiagen, Hilden, Germany), briefly agitated, and left for $10 \mathrm{~min}$ at room temperature. After centrifugation at $8,000 \mathrm{rpm}$, the supernatant was removed and frozen with liquid nitrogen.

Measurements of extracellular DNA in planktonic culture. Culture samples of $P$. aeruginosa PA14 were cultivated and treated with ciprofloxacin, novobiocin, or CN-DM-861 as described in the section above.

To quantify the amount of extracellular DNA (eDNA) in planktonic cell culture, we employed the method described by Allesen-Holm et al. (38). After reaching the target OD of 0.6, cultures were centrifuged ( $3 \mathrm{~min}, 10,000 \mathrm{rpm}$ ) and the supernatant was transferred to a new Eppendorf tube. $\mathrm{NaCl}$ solution $(2.5 \mathrm{M})$ was added to the supernatant to a concentration of $0.25 \mathrm{M}$. After vortexing, a 2:1 volume of ethanol was added to precipitate the DNA. After vortexing and standing for $5 \mathrm{~min}$, the suspension was centrifuged $(10 \mathrm{~min}, 16,000 \mathrm{rpm})$ and the supernatant was removed with a pipette. The residue (eDNA) was dissolved in Tris-EDTA (TE) buffer and thoroughly vortexed. The amount of eDNA was quantified by measuring the $\mathrm{OD}_{260}$ and using TE buffer as a reference. Four independent cultures (biological replicates) were used for each treatment.

Metabolomics extraction and LC-MS analysis. For the analysis of the intracellular metabolome, cell pellets were extracted with $500 \mu \mathrm{l} 80 \%$ methanol containing caffeine $(0.1 \mathrm{mg} /$ liter), nortriptyline $(0.1 \mathrm{mg} / \mathrm{liter})$, glipizide $(0.3 \mathrm{mg} / \mathrm{liter})$, and naproxen $(0.8 \mathrm{mg} / \mathrm{liter})$ as internal standards. Extraction was achieved through shaking vigorously for $5 \mathrm{~min}$ and sonication in an ultrasonic bath for $20 \mathrm{~min}$ at $0^{\circ} \mathrm{C}$. After centrifugation $\left(13,000 \mathrm{rpm}, 4^{\circ} \mathrm{C}\right), 400 \mu \mathrm{l}$ of the supernatant was concentrated to dryness using a SpeedVac and resuspended in $40 \mu \mathrm{l}$ acetonitrile/water (1:1, containing $0.1 \%$ [vol/vol] formic acid) using ultrasonication for dissolving the residue.

For each sample, $1 \mu$ l was analyzed by reversed phase ultrahigh-performance liquid chromatography coupled to quadrupole time-of-flight mass spectrometry. In addition, a pool sample was prepared by mixing $5 \mu$ l of each sample.

The samples were separated using ultra high-performance liquid chromatography, performed on a Dionex Ultimate 3000 UPLC system (Thermo Fisher Scientific, Waltham, MA) using a 150 by $2.1 \mathrm{~mm}$ Kinetex $C_{18}$ column with 1.7- $\mu \mathrm{m}$ particle size (Phenomenex, Aschaffenburg, Germany) column with a flow rate of $300 \mu \mathrm{l} / \mathrm{min}$.

Gradient elution with water with $0.1 \%$ (vol/vol) formic acid as eluent $A$ and acetonitrile with $0.1 \%$ ( $\mathrm{vol} / \mathrm{vol}$ ) formic acid as eluent B was run as follows: $1 \% \mathrm{~B}$ for $t=0 \mathrm{~min}$ to $t=2 \mathrm{~min}$, linear gradient from $1 \% \mathrm{~B}$ to $100 \% \mathrm{~B}$ from $t=2 \mathrm{~min}$ to $t=20 \mathrm{~min}$, hold $100 \% \mathrm{~B}$ until $t=25 \mathrm{~min}$, and linear gradient from $100 \%$ $\mathrm{B}$ to $1 \% \mathrm{~B}$ from $t=25 \mathrm{~min}$ to $t=30 \mathrm{~min}$.

The samples were analyzed by positive mode electrospray ionization quadrupole time-of-flight mass spectrometry on a maXis HD QTOF (Bruker, Bremen, Germany) in full scan mode (50 to 1,500 Da). Accurate masses were obtained by internal calibration using an ion cluster of sodium formate and lock mass calibration. The pooled sample was analyzed using data-dependent MS/MS by collision-induced dissociation of the three most abundant ions in each scan, making use of Bruker's "Smart Exclusion" functionality to minimize multiple fragmentation of the same ion. 
RNA isolation and library preparation and sequencing. The bacterial suspensions treated with RNAprotect (Qiagen) were stored at $-80^{\circ} \mathrm{C}$. Libraries for transcriptomics were generated according to references 64 and 65.

RNA samples were quality checked by the use of the RNA Nano kit with an Agilent Bioanalyzer 2100 (Agilent Technologies, CA, USA). To remove rRNA, the Ribo-Zero bacteria kit (Illumina, CA, USA) was used. Samples were sequenced in paired-end mode on an Illumina NovaSeq 6000 device $(2 \times 50 \mathrm{bp})$.

Bioinformatics processing of metabolomics data. LC-MS data generated with the Bruker maXis $\mathrm{HD}$ mass spectrometer were converted to the $\mathrm{mzXML}$ format from the Bruker centroid data using Bruker Compass Xport. Preprocessing of the mzXML raw files was carried out using XCMS (version 3.4.4) (66) as follows.

For peak picking, the centWave algorithm was employed with parameter settings peakwith from 5 to $25 \mathrm{~s}, \mathrm{ppm}=10$, snthresh $=100, \operatorname{mzdiff}=0.01$, prefilter $=c(2,1000)$, noise $=100$. For chromatogram alignment, the obiwarp algorithm was used with default settings, and grouping of peaks from different samples was achieved by using the group.density function with settings bw $=5, \mathrm{mzwid}=0.015$, $\operatorname{minfrac}=0.5$, minsamp $=1$.

Feature annotation (isotopic features and adduct formations, dimers, multimers, neutral losses) was carried out using the CAMERA package (version 1.38.1) (67) with parameters perfwhm $=0.6$, mzabs $=0.01$, cor_eic_th $=0.75$.

Further processing of the peak table employed an in-house script involving the following steps. A retention time filter (features with a retention time lower than $50 \mathrm{~s}$ or higher than 1,200 s were removed from the peak table) was employed to get rid of the sodium formate cluster peak and the signals arising from impurities that elute during washing of the column with $100 \%$ acetonitrile. To account for experimental variability, peak areas were normalized using the internal standard nortriptyline. After removal of all features belonging to internal standards, the data for each sample were further normalized through the use of the OD at harvest of the respective culture as a proxy for cell number. Missing value imputation was carried out using the imputeRowMinRand function of the XCMS package, which replaces missing values with random numbers from a normal distribution based on the row minimum. For metabolite identification, tandem mass spectra recorded from a pooled sample were compared to an in-house database of spectra from authentic standards and/or metabolite databases using the Bruker DataAnalysis software (version 4.0, for results see Tables S1 and S3). To perform hierarchical clustering, the data set was scaled, Euclidean distance was utilized as distance measure, and Ward's method was utilized as linkage criterion. For PCA, the data set was scaled and centered. To identify significantly abundant features, Welch's $t$ test was used and a threshold of $\log _{2}$ (fold change) of $\log _{2}(1.5)$ or greater and of $-\log _{2}(1.5)$ or lower, respectively, with a $P$ value of $<0.05$ was applied. The raw data are deposited as MassIVE data set MSV000086820 (https://massive.ucsd.edu/). The R scripts used for preprocessing and analyzing the data are deposited in a GitHub archive: https://github.com/raimofranke/PA14_gyr_inhibitors.

Bioinformatics processing of transcriptomics data. Short reads were aligned to the UCBPP-PA14 reference genome (NC_008463, available for download from the Pseudomonas genome database, http:// pseudomonas.com) using the subread algorithm (68) implemented in R (R version 3.5.3, Foundation for Statistical Computing, Vienna, Austria; Rsubread version 1.32.4) (69). Count estimation was performed using the featureCounts function (70) as implemented in Rsubread. Differential gene expression analysis was performed using the $R$ package edgeR (version 3.24.3) (71). Trimmed mean of M-values (TMM) normalization was performed, followed by quasi-likelihood F-test to test for significant differential expression in each gene (glmQLFTest-function). To correct for multiple testing, the Benjamini Hochberg procedure was used to calculate the false discovery rate (FDR). For the identification of differentially expressed genes between gyrase inhibitor treatment and untreated control, a threshold of $\log _{2}$ (fold change) of 1 or greater and of -1 or lower, respectively, with FDR of $<0.05$ was applied. For clustering and PCA, moderated log counts per million (log CPM) were calculated using the CPM function. For PCA, centered log CPM data were used. For hierarchical clustering, the log CPM data were used with Euclidean distance as distance measure and Ward's method as linkage criterion. For annotation of the results table (Table S2) with KEGG pathway, GO term, and pseudoCAP class information, the annotator functionality of the BACTOME database was used (29).

Data and code availability. The RNA-Seq data generated during this study have been deposited in NCBI's Gene Expression Omnibus (72) and are accessible through GEO series accession number GSE166602. The metabolomics data have been deposited in the MassIVE database (https://massive.ucsd .edu/) under accession number MSV000086820. The R scripts used for processing the RNA-Seq, metabolomics data, and generation of figures have been deposited in a GitHub archive: https://github.com/ raimofranke/PA14_gyr_inhibitors.

\section{SUPPLEMENTAL MATERIAL}

Supplemental material is available online only.

FIG S1, TIF file, 2.4 MB.

TABLE S1, DOCX file, $0.02 \mathrm{MB}$.

TABLE S2, XLSX file, 1.5 MB.

TABLE S3, XLSX file, 0.5 MB. 


\section{ACKNOWLEDGMENTS}

We thank Ulrike Beutling and Astrid Dröge for technical assistance regarding LCMS/MS measurements and RNA-Seq library preparation, respectively, and we thank Giambattista Testolin and Antje Ritter for providing the cystobactamid derivatives. The Genome Analytics group of the Helmholtz Center for Infection Research is acknowledged for providing Illumina sequencing services.

R.F. designed the experiments, performed the bioinformatics data processing, interpreted the data, and wrote the manuscript, H.O. conducted all the experiments, S.H. provided technical support and reagents for the RNA-Seq analysis, and M.B. designed and supervised the project, interpreted the data, and wrote the manuscript.

M.B. has filed a patent application on cystobactamids as antibiotics (WO 2019038405).

\section{REFERENCES}

1. World Health Organization. 2019. No time to wait: securing the future from drug-resistant infections.

2. Theuretzbacher U, Gottwalt S, Beyer P, Butler M, Czaplewski L, Lienhardt C, Moja L, Paul M, Paulin S, Rex JH, Silver LL, Spigelman M, Thwaites GE, Paccaud JP, Harbarth S. 2019. Analysis of the clinical antibacterial and antituberculosis pipeline. Lancet Infect Dis 19:e40-e50. https://doi.org/10 .1016/S1473-3099(18)30513-9.

3. Stokes JM, Lopatkin AJ, Lobritz MA, Collins JJ. 2019. Bacterial metabolism and antibiotic efficacy. Cell Metab 30:251-259. https://doi.org/10.1016/j .cmet.2019.06.009.

4. Chernov VM, Chernova OA, Mouzykantov AA, Lopukhov LL, Aminov RI. 2019. Omics of antimicrobials and antimicrobial resistance. Expert Opin Drug Discov 14:455-468. https://doi.org/10.1080/17460441.2019.1588880.

5. Hoerr V, Duggan GE, Zbytnuik L, Poon KKH, Große C, Neugebauer U, Methling K, Löffler B, Vogel HJ. 2016. Characterization and prediction of the mechanism of action of antibiotics through NMR metabolomics. BMC Microbiol 16:82. https://doi.org/10.1186/s12866-016-0696-5.

6. Vincent IM, Ehmann DE, Mills SD, Perros M, Barrett MP. 2016. Untargeted metabolomics to ascertain antibiotic modes of action. Antimicrob Agents Chemother 60:2281-2291. https://doi.org/10.1128/AAC.02109-15.

7. Zampieri M, Zimmermann M, Claassen M, Sauer U. 2017. Nontargeted metabolomics reveals the multilevel response to antibiotic perturbations. Cell Rep 19:1214-1228. https://doi.org/10.1016/j.celrep.2017.04.002.

8. Han ML, Zhu Y, Creek DJ, Lin YW, Gutu AD, Hertzog P, Purcell T, Shen HH, Moskowitz SM, Velkov T, Li J. 2019. Comparative metabolomics and transcriptomics reveal multiple pathways associated with polymyxin killing in Pseudomonas aeruginosa. mSystems 4:e00149-18. https://doi.org/10 $.1128 / \mathrm{mSystems.00149-18.}$

9. Zampieri M, Szappanos B, Buchieri MV, Trauner A, Piazza I, Picotti P, Gagneux S, Borrell S, Gicquel B, Lelievre J, Papp B, Sauer U. 2018. Highthroughput metabolomic analysis predicts mode of action of uncharacterized antimicrobial compounds. Sci Transl Med 10:eaal3973. https://doi .org/10.1126/scitransImed.aal3973.

10. Ziegler S, Pries V, Hedberg C, Waldmann H. 2013. Target identification for small bioactive molecules: finding the needle in the haystack. Angew Chem Int Ed Engl 52:2744-2792. https://doi.org/10.1002/anie.201208749.

11. Kapoor S, Waldmann H, Ziegler S. 2016. Novel approaches to map small molecule-target interactions. Bioorg Med Chem 24:3232-3245. https:// doi.org/10.1016/j.bmc.2016.05.020.

12. Tacconelli E, Carrara E, Savoldi A, Harbarth S, Mendelson M, Monnet DL, Pulcini C, Kahlmeter G, Kluytmans J, Carmeli Y, Ouellette M, Outterson K, Patel J, Cavaleri M, Cox EM, Houchens CR, Grayson ML, Hansen P, Singh N, Theuretzbacher U, Magrini N, Group WHOPPLW. 2018. Discovery, research, and development of new antibiotics: the WHO priority list of antibiotic-resistant bacteria and tuberculosis. Lancet Infect Dis 18:318-327. https://doi.org/10.1016/S1473-3099(17)30753-3.

13. Malhotra S, Hayes D, Jr, Wozniak DJ. 2019. Cystic fibrosis and Pseudomonas aeruginosa: the host-microbe interface. Clin Microbiol Rev 32:e0013818. https://doi.org/10.1128/CMR.00138-18.

14. Morita Y, Tomida J, Kawamura Y. 2014. Responses of Pseudomonas aeruginosa to antimicrobials. Front Microbiol 4:422. https://doi.org/10.3389/ fmicb.2013.00422.

15. Linares JF, Gustafsson I, Baquero F, Martinez JL. 2006. Antibiotics as intermicrobial signaling agents instead of weapons. Proc Natl Acad Sci U S A 103:19484-19489. https://doi.org/10.1073/pnas.0608949103.
16. Cirz RT, O'Neill BM, Hammond JA, Head SR, Romesberg FE. 2006. Defining the Pseudomonas aeruginosa SOS response and its role in the global response to the antibiotic ciprofloxacin. J Bacteriol 188:7101-7110. https://doi.org/10.1128/JB.00807-06.

17. Murray JL, Kwon T, Marcotte EM, Whiteley M. 2015. Intrinsic antimicrobial resistance determinants in the superbug Pseudomonas aeruginosa. $\mathrm{mBio}$ 6:e01603-15. https://doi.org/10.1128/mBio.01603-15.

18. Baumann S, Herrmann J, Raju R, Steinmetz H, Mohr KI, Huttel S, Harmrolfs K, Stadler M, Muller R. 2014. Cystobactamids: myxobacterial topoisomerase inhibitors exhibiting potent antibacterial activity. Angew Chem Int Ed Engl 53:14605-14609. https://doi.org/10.1002/anie.201409964.

19. Huttel S, Testolin G, Herrmann J, Planke T, Gille F, Moreno M, Stadler M, Bronstrup M, Kirschning A, Muller R. 2017. Discovery and total synthesis of natural cystobactamid derivatives with superior activity against Gramnegative pathogens. Angew Chem Int Ed Engl 56:12760-12764. https:// doi.org/10.1002/anie.201705913.

20. Elgaher WAM, Hamed MM, Baumann S, Herrmann J, Siebenburger L, Krull J, Cirnski K, Kirschning A, Bronstrup M, Muller R, Hartmann RW. 2020. Cystobactamid 507: concise synthesis, mode of action, and optimization toward more potent antibiotics. Chemistry 26:7219-7225. https://doi.org/ 10.1002/chem.202000117.

21. Testolin G, Cirnski K, Rox K, Prochnow H, Fetz V, Grandclaudon C, Mollner T, Baiyoumy A, Ritter A, Leitner C, Krull J, van den Heuvel J, Vassort A, Sordello S, Hamed MM, Elgaher WAM, Herrmann J, Hartmann RW, Müller R, Brönstrup M. 2020. Synthetic studies of cystobactamids as antibiotics and bacterial imaging carriers lead to compounds with high in vivo efficacy. Chem Sci 11:1316-1334. https://doi.org/10.1039/C9SC04769G.

22. Planke T, Cirnski K, Herrmann J, Müller R, Kirschning A. 2020. Synthetic and biological studies on new urea and triazole containing cystobactamid derivatives. Chemistry 26:4289-4296. https://doi.org/10.1002/chem .201904073.

23. Vanden Broeck A, McEwen AG, Chebaro Y, Potier N, Lamour V. 2019. Structural basis for DNA gyrase interaction with coumermycin A1. J Med Chem 62:4225-4231. https://doi.org/10.1021/acs.jmedchem.8b01928.

24. Ali JA, Jackson AP, Howells AJ, Maxwell A. 1993. The 43-kilodalton N-terminal fragment of the DNA gyrase B protein hydrolyzes ATP and binds coumarin drugs. Biochemistry 32:2717-2724. https://doi.org/10.1021/ bi00061a033.

25. Collin F, Karkare S, Maxwell A. 2011. Exploiting bacterial DNA gyrase as a drug target: current state and perspectives. Appl Microbiol Biotechnol 92:479-497. https://doi.org/10.1007/s00253-011-3557-z.

26. Brazas MD, Hancock RE. 2005. Ciprofloxacin induction of a susceptibility determinant in Pseudomonas aeruginosa. Antimicrob Agents Chemother 49:3222-3227. https://doi.org/10.1128/AAC.49.8.3222-3227.2005.

27. Li A, Mao D, Yoshimura A, Rosen PC, Martin WL, Gallant E, Wuhr M, Seyedsayamdost MR. 2020. Multi-omic analyses provide links between low-dose antibiotic treatment and induction of secondary metabolism in Burkholderia thailandensis. mBio 11:e03210-19. https://doi.org/10.1128/ mBio.03210-19.

28. Qin TT, Kang HQ, Ma P, Li PP, Huang LY, Gu B. 2015. SOS response and its regulation on the fluoroquinolone resistance. Ann Transl Med 3:358. https://doi.org/10.3978/j.issn.2305-5839.2015.12.09.

29. Hornischer K, Khaledi A, Pohl S, Schniederjans M, Pezoldt L, Casilag F, Muthukumarasamy U, Bruchmann S, Thoming J, Kordes A, Haussler S. 2019. BACTOME-a reference database to explore the sequence- and gene 
expression-variation landscape of Pseudomonas aeruginosa clinical isolates. Nucleic Acids Res 47:D716-D720. https://doi.org/10.1093/nar/ gky895.

30. Kohanski MA, Dwyer DJ, Hayete B, Lawrence CA, Collins JJ. 2007. A common mechanism of cellular death induced by bactericidal antibiotics. Cell 130:797-810. https://doi.org/10.1016/j.cell.2007.06.049.

31. Suzuki YJ, Carini M, Butterfield DA. 2010. Protein carbonylation. Antioxid Redox Signal 12:323-325. https://doi.org/10.1089/ars.2009.2887.

32. Chang W, Small DA, Toghrol F, Bentley WE. 2005. Microarray analysis of Pseudomonas aeruginosa reveals induction of pyocin genes in response to hydrogen peroxide. BMC Genomics 6:115. https://doi.org/10.1186/ 1471-2164-6-115.

33. Sun Z, Shi J, Liu C, Jin Y, Li K, Chen R, Jin S, Wu W. 2014. PrtR homeostasis contributes to Pseudomonas aeruginosa pathogenesis and resistance against ciprofloxacin. Infect Immun 82:1638-1647. https://doi.org/10 .1128/IAI.01388-13.

34. Oluyombo O, Penfold CN, Diggle SP. 2019. Competition in biofilms between cystic fibrosis isolates of Pseudomonas aeruginosa is shaped by R-pyocins. mBio 10:e01828-18. https://doi.org/10.1128/mBio.01828-18.

35. Coleman SR, Blimkie T, Falsafi R, Hancock REW. 2020. Multidrug adaptive resistance of Pseudomonas aeruginosa swarming cells. Antimicrob Agents Chemother 64:e02182-20. https://doi.org/10.1128/AAC.01999-19.

36. Turnbull L, Toyofuku M, Hynen AL, Kurosawa M, Pessi G, Petty NK, Osvath SR, Carcamo-Oyarce G, Gloag ES, Shimoni R, Omasits U, Ito S, Yap X, Monahan LG, Cavaliere R, Ahrens CH, Charles IG, Nomura N, Eberl L, Whitchurch CB. 2016. Explosive cell lysis as a mechanism for the biogenesis of bacterial membrane vesicles and biofilms. Nat Commun 7:11220. https://doi.org/10.1038/ncomms11220.

37. McFarland KA, Dolben EL, LeRoux M, Kambara TK, Ramsey KM, Kirkpatrick RL, Mougous JD, Hogan DA, Dove SL. 2015. A self-lysis pathway that enhances the virulence of a pathogenic bacterium. Proc Natl Acad Sci U S A 112:8433-8438. https://doi.org/10.1073/pnas.1506299112.

38. Allesen-Holm M, Barken KB, Yang L, Klausen M, Webb JS, Kjelleberg S, Molin S, Givskov M, Tolker-Nielsen T. 2006. A characterization of DNA release in Pseudomonas aeruginosa cultures and biofilms. Mol Microbiol 59:1114-1128. https://doi.org/10.1111/j.1365-2958.2005.05008.x.

39. Moradali MF, Ghods S, Rehm BH. 2017. Pseudomonas aeruginosa lifestyle: a paradigm for adaptation, survival, and persistence. Front Cell Infect Microbiol 7:39. https://doi.org/10.3389/fcimb.2017.00039.

40. Pierson LS, 3rd, Pierson EA. 2010. Metabolism and function of phenazines in bacteria: impacts on the behavior of bacteria in the environment and biotechnological processes. Appl Microbiol Biotechnol 86:1659-1670. https://doi.org/10.1007/s00253-010-2509-3.

41. Wang Y, Wilks JC, Danhorn T, Ramos I, Croal L, Newman DK. 2011. Phenazine-1-carboxylic acid promotes bacterial biofilm development via ferrous iron acquisition. J Bacteriol 193:3606-3617. https://doi.org/10.1128/ JB.00396-11.

42. Swanson BL, Hager $P$, Phibbs $P$, Jr, Ochsner $U$, Vasil ML, Hamood AN. 2000. Characterization of the 2-ketogluconate utilization operon in Pseudomonas aeruginosa PAO1. Mol Microbiol 37:561-573. https://doi.org/10 .1046/j.1365-2958.2000.02012.x.

43. Daddaoua A, Fillet S, Fernandez M, Udaondo Z, Krell T, Ramos JL. 2012. Genes for carbon metabolism and the ToxA virulence factor in Pseudomonas aeruginosa are regulated through molecular interactions of PtxR and PtxS. PLoS One 7:e39390. https://doi.org/10.1371/journal.pone.0039390.

44. del Castillo T, Ramos JL, Rodriguez-Herva JJ, Fuhrer T, Sauer U, Duque E. 2007. Convergent peripheral pathways catalyze initial glucose catabolism in Pseudomonas putida: genomic and flux analysis. J Bacteriol 189:5142-5152. https://doi.org/10.1128/JB.00203-07.

45. Raneri M, Pinatel E, Peano C, Rampioni G, Leoni L, Bianconi I, Jousson O, Dalmasio C, Ferrante P, Briani F. 2018. Pseudomonas aeruginosa mutants defective in glucose uptake have pleiotropic phenotype and altered virulence in non-mammal infection models. Sci Rep 8:16912. https://doi.org/ 10.1038/s41598-018-35087-y.

46. Kohlstedt M, Wittmann C. 2019. GC-MS-based (13)C metabolic flux analysis resolves the parallel and cyclic glucose metabolism of Pseudomonas putida KT2440 and Pseudomonas aeruginosa PAO1. Metab Eng 54:35-53. https://doi.org/10.1016/j.ymben.2019.01.008.

47. Brochado AR, Telzerow A, Bobonis J, Banzhaf M, Mateus A, Selkrig J, Huth E, Bassler S, Zamarreño Beas J, Zietek M, Ng N, Foerster S, Ezraty B, Py B, Barras F, Savitski MM, Bork P, Göttig S, Typas A. 2018. Species-specific activity of antibacterial drug combinations. Nature 559:259-263. https://doi .org/10.1038/s41586-018-0278-9.
48. Schroder W, Goerke C, Wolz C. 2013. Opposing effects of aminocoumarins and fluoroquinolones on the SOS response and adaptability in Staphylococcus aureus. J Antimicrob Chemother 68:529-538. https://doi.org/10 $.1093 / \mathrm{jac} / \mathrm{dks} 456$.

49. Smith CL. 1983. recF-dependent induction of recA synthesis by coumermycin, a specific inhibitor of the B subunit of DNA gyrase. Proc Natl Acad Sci U S A 80:2510-2513. https://doi.org/10.1073/pnas.80.9.2510.

50. Osburne MS, Zavodny SM, Peterson GA. 1988. Drug-induced relaxation of supercoiled plasmid DNA in Bacillus subtilis and induction of the SOS response. J Bacteriol 170:442-445. https://doi.org/10.1128/jb.170 .1.442-445.1988.

51. Martis BS, Forquet R, Reverchon S, Nasser W, Meyer S. 2019. DNA supercoiling: an ancestral regulator of gene expression in pathogenic bacteria? Comput Struct Biotechnol J 17:1047-1055. https://doi.org/10.1016/j.csbj 2019.07.013.

52. Dorman CJ. 2019. DNA supercoiling and transcription in bacteria: a twoway street. BMC Mol Cell Biol 20:26. https://doi.org/10.1186/s12860-019 $-0211-6$.

53. Blot N, Mavathur R, Geertz M, Travers A, Muskhelishvili G. 2006. Homeostatic regulation of supercoiling sensitivity coordinates transcription of the bacterial genome. EMBO Rep 7:710-715. https://doi.org/10.1038/sj .embor.7400729.

54. Parsa JY, Ramachandran S, Zaheen A, Nepal RM, Kapelnikov A, Belcheva A, Berru M, Ronai D, Martin A. 2012. Negative supercoiling creates single-stranded patches of DNA that are substrates for AID-mediated mutagenesis. PLoS Genet 8:e1002518. https://doi.org/10.1371/journal .pgen.1002518.

55. Moreau PL. 1987. Effects of overproduction of single-stranded DNA-binding protein on RecA protein-dependent processes in Escherichia coli. J Mol Biol 194:621-634. https://doi.org/10.1016/0022-2836(87)90239-7.

56. Mestres J, Gregori-Puigjane E, Valverde S, Sole RV. 2008. Data completeness-the Achilles heel of drug-target networks. Nat Biotechnol 26:983-984. https://doi.org/10.1038/nbt0908-983.

57. Wright MH, Sieber SA. 2016. Chemical proteomics approaches for identifying the cellular targets of natural products. Nat Prod Rep 33:681-708. https://doi.org/10.1039/C6NP00001K.

58. Eirich J, Orth R, Sieber SA. 2011. Unraveling the protein targets of vancomycin in living S. aureus and E. faecalis cells. J Am Chem Soc 133:12144-12153. https://doi.org/10.1021/ja2039979.

59. Marcu MG, Chadli A, Bouhouche I, Catelli M, Neckers LM. 2000. The heat shock protein 90 antagonist novobiocin interacts with a previously unrecognized ATP-binding domain in the carboxyl terminus of the chaperone. J Biol Chem 275:37181-37186. https://doi.org/10.1074/jbc.M003701200.

60. Penterman J, Singh PK, Walker GC. 2014. Biological cost of pyocin production during the SOS response in Pseudomonas aeruginosa. J Bacteriol 196:3351-3359. https://doi.org/10.1128/JB.01889-14.

61. Erdmann J, Preusse M, Khaledi A, Pich A, Haussler S. 2018. Environmentdriven changes of mRNA and protein levels in Pseudomonas aeruginosa. Environ Microbiol 20:3952-3963. https://doi.org/10.1111/1462-2920.14419.

62. Ciofu O, Tolker-Nielsen T. 2019. Tolerance and resistance of Pseudomonas aeruginosa biofilms to antimicrobial agents-how $\mathrm{P}$ aeruginosa can escape antibiotics. Front Microbiol 10:913. https://doi.org/10.3389/fmicb.2019 .00913.

63. Lambert RJ, Pearson J. 2000. Susceptibility testing: accurate and reproducible minimum inhibitory concentration (MIC) and non-inhibitory concentration (NIC) values. J Appl Microbiol 88:784-790. https://doi.org/10 .1046/j.1365-2672.2000.01017.x.

64. Shishkin AA, Giannoukos G, Kucukural A, Ciulla D, Busby M, Surka C, Chen J, Bhattacharyya RP, Rudy RF, Patel MM, Novod N, Hung DT, Gnirke A, Garber M, Guttman M, Livny J. 2015. Simultaneous generation of many RNA-seq libraries in a single reaction. Nat Methods 12:323-325. https:// doi.org/10.1038/nmeth.3313.

65. Bhattacharyya RP, Bandyopadhyay N, Ma P, Son SS, Liu J, He LL, Wu L, Khafizov R, Boykin R, Cerqueira GC, Pironti A, Rudy RF, Patel MM, Yang R, Skerry J, Nazarian E, Musser KA, Taylor J, Pierce VM, Earl AM, Cosimi LA, Shoresh N, Beechem J, Livny J, Hung DT. 2019. Simultaneous detection of genotype and phenotype enables rapid and accurate antibiotic susceptibility determination. Nat Med 25:1858-1864. https://doi.org/10.1038/ s41591-019-0650-9.

66. Smith CA, Want EJ, O'Maille G, Abagyan R, Siuzdak G. 2006. XCMS: processing mass spectrometry data for metabolite profiling using nonlinear peak alignment, matching, and identification. Anal Chem 78:779-787. https://doi.org/10.1021/ac051437y. 
67. Kuhl C, Tautenhahn R, Bottcher C, Larson TR, Neumann S. 2012. CAMERA: an integrated strategy for compound spectra extraction and annotation of liquid chromatography/mass spectrometry data sets. Anal Chem 84:283-289. https://doi.org/10.1021/ac202450g.

68. Liao Y, Smyth GK, Shi W. 2013. The Subread aligner: fast, accurate and scalable read mapping by seed-and-vote. Nucleic Acids Res 41: e108-e108. https://doi.org/10.1093/nar/gkt214.

69. Liao Y, Smyth GK, Shi W. 2019. The R package Rsubread is easier, faster, cheaper and better for alignment and quantification of RNA sequencing reads. Nucleic Acids Res 47:e47-e47. https://doi.org/10.1093/nar/ gkz114.

70. Liao Y, Smyth GK, Shi W. 2014. featureCounts: an efficient general purpose program for assigning sequence reads to genomic features. Bioinformatics 30:923-930. https://doi.org/10.1093/bioinformatics/ btt656.
71. Robinson MD, McCarthy DJ, Smyth GK. 2010. edgeR: a Bioconductor package for differential expression analysis of digital gene expression data. Bioinformatics 26:139-140. https://doi.org/10.1093/bioinformatics/ btp616.

72. Edgar R, Domrachev M, Lash AE. 2002. Gene Expression Omnibus: NCBI gene expression and hybridization array data repository. Nucleic Acids Res 30:207-210. https://doi.org/10.1093/nar/30.1.207.

73. Sun W, Alexander T, Man Z, Xiao F, Cui F, Qi X. 2018. Enhancing 2-ketogluconate production of Pseudomonas plecoglossicida JUIM01 by maintaining the carbon catabolite repression of 2-ketogluconate metabolism. Molecules 23:2629. https://doi.org/10.3390/molecules23102629.

74. Udaondo Z, Ramos JL, Segura A, Krell T, Daddaoua A. 2018. Regulation of carbohydrate degradation pathways in Pseudomonas involves a versatile set of transcriptional regulators. Microb Biotechnol 11:442-454. https:// doi.org/10.1111/1751-7915.13263. 